\title{
Management of Distal Femoral Fractures: a Comparative Study between Open Reduction and Internal Fixation by Distal Femur Locked Plate and Retrograde Nailing \\ Mohamed Atef Abd-Elhamid Ismail ${ }^{1, *}$ M.B.B.Ch, Adnan A .Alim Al sebaie ${ }^{2}$ MD., Ahmed Ismail Hamoda $^{2}$ MD
}

\author{
* Corresponding Author: \\ Mohamed Atef Abd-Elhamid \\ Cap.matef91@gmail.com
}

Received for publication August 11, 2020; Accepted January 3, 2020; Published online January 3, 2020.

Copyright 2020 The Authors published by Al-Azhar University, Faculty of Medicine, Cairo, Egypt. All rights reserved. This an openaccess article distributed under the legal terms, where it is permissible to download and share the work provided it is properly cited. The work cannot be changed in any way or used commercially.

doi: $10.21608 /$ aimj.2021.35593.1284

${ }^{1}$ El-Helmeya Military hospital for orthopedic and reconstructive surgery

${ }^{2}$ Department of Orthopedic Surgery, Faculty of Medicine, Al-Azhar University, Egypt

\begin{abstract}
Background: Fractures of the distal femur are usually rare and severe , The usual context is a high energy trauma in a young patient and a domestic accident in an elderly person. There are different modalities of management of distal femur fractures among which are the retrograde intramedullary nail and locked compression plate. Both operative stabilizing systems follow the principle of biological osteosynthesis.

Aim of the work: To evaluate and compare the outcome of each method of fixation of distal femur fractures as regard; operative time; early mobilization; knee range of motion; time of healing and complications.

Materials and methods: This study was conducted on 32 patients attending to El-Helmeya Military Hospital, and Sayed Galal University Hospitals, for comparison between fixation of distal femoral fractures using femoral retrograde intramedullary nails $(\mathrm{RN})$ and distal femoral locked compression plats (LP). The study was conducted between September 2019 and May 2020, The series included 16 cases treated with femoral retrograde interlocking nails (RN) and 16 cases treated with distal femoral locked compression plats (LP).

Results: The retrograde nail was superior to locked plate in the aspect of operative time with less operative blood loss and less time to union.

Conclusion: Based on this study; accepted outcome had been achieved with both methods compared with results of previous studies. However in our series nail showed better union rate and less surgical morbidities.

Keywords: Distal femur fracture; retrograde nail; locked plate.

Disclosure: The authors have no financial interest to declare in relation to the content of this article. The Article Processing Charge was paid for by the authors.

Authorship: All authors have a substantial contribution to the article.
\end{abstract}


or alignment using one versus the other. This study aims to elucidate the respective advantages and disadvantages of each technique in a randomized clinical trial. Radiologic and functional outcomes will be collected and compared. This will help orthopedic surgeons to decide the ultimate treatment for patients with this injury.

\section{MATERIALS AND METHODS}

Study design:- This study is a prospective study, consisted of 32 patients who were subjected for comparison between fixation of distal femoral fractures using femoral retrograde intramedullary nails (RN) and distal femoral locked compression plates (LP). The study was conducted between September 2019 and May 2020.This study has been executed at the orthopedic Surgery Department, El-Helmeya Military Hospital, and Sayed Galal University Hospital (Bab EL.Sharia hospital), Faculty of Medicine, Al-Azhar University, Cairo, Egypt.

Inclusion criteria:- Skeletally mature patients; Fracture of the distal femur with or without intraarticular extension (AO/OTA Types A1-2-3 and C12) ; Fracture requiring operative treatment amenable to either Retrograde Nail or distal femur locked plate and Informed consent obtained.

Exclusion Criteria:- Skeletally immature patients; Open fractures; Associated vascular injury; Pathological fractures ; Peri-prosthetic fractures; Associated ligamentous injuries of the knee and Medically unfit.

Patients who met the criteria above were divided into two groups: Group A: were treated by retrograde nail (RN); Group B: were treated by distal femur locked plate (LP).

Ethical approval:- An approved was obtained from the Ethical Research Board (ERB) of the Faculty of Medicine, Al-Azhar University, Cairo, Egypt. Prior to study proceeding, all patients assigned informed consents after the obvious explanation of the possible adverse events.

Statistical analysis of the data:-

Statistical presentation and analysis of the present study was conducted, using the mean standard Deviation; unpaired student t-test and chi-square tests by SPSS V20. Unpaired Student T-test was used to compare between two groups in quantitative data .Chi-square, contingency table and fisher exact test were used to detect relation between different qualitative variables. Significance level: NonSignificant $>0.05$; Significant $<0.05 ;$ High Significant $<0.001$

\section{RESULTS}

This is a prospective randomized control study aiming to compare between two fixation methods for distal femoral fractures; femoral Retrograde
Intramedullary Nail and locked plate. The study was done in El-Helmeya Military Hospital and, Sayed Galal University Hospitals (Bab EL.Sharia hospital), from June 2019 to May 2020 (date of last follow up).

The study was conducted on 32 patients, 16 cases treated with Femoral Retrograde Interlocking Nails and 16 cases treated with distal femur locked plate.

The AO/OTA classification was used to grade the fractures. In the Retrograde Nail femur group; there were 15 type A fractures $(93.75 \%)$, and 1 type C fractures $(6.25 \%)$. In the LP group; there were 9 type A fractures $(56.25 \%)$, and 7 type $\mathrm{C}$ fractures (43.75\%). In RN (group); the mean operative time was 93.56 minutes.(range from 72 to 125 minutes); while In LP (group); the mean operative time was 107.75 minutes. (range from 94 to 130 minutes). (Table 1). In RIN group; the estimated mean blood loss $248.125 \mathrm{cc}$ (range, 100 to-600 cc); while In LP group; the estimated mean blood loss $434.375 \mathrm{cc}$ (range, 100-700 cc). (Table 2). Mean time till union in $\mathrm{RN}$ group was 11.4 weeks while in LP group was 15.8 weeks. (Table 3 ). In RN group; there was 8 cases had No difficulty, 4 cases had Little bit of difficulty, 2 cases had Moderate difficulty, 1 cases had Quite bit of difficulty and 1 case had Extreme difficulty or unable to perform activity, while in the LP group there is 6 cases had No difficulty, 5 cases had Little bit of difficulty, 2 cases had Moderate difficulty , 2 cases had Quite bit of difficulty and 1 case had Extreme difficulty or unable to perform activity. In RN group; there was 15 cases with more than 100 o knee flexion, 1 case with 90 o -100 o flexion, while in the LP group; there was 14 cases with more than 100 o knee flexion, 1 case with 90 o 100o flexion and 1 case with $70 \mathrm{o}-89 \mathrm{o}$ flexion. Complication that was recorded in both method of fixation, as follow, Infection; In RN group : no cases of infection ; In LP group : one case was complicated by superficial infection in the early postoperative period which was managed by debridement and antibiotics according to culture and sensitivity leading to complete resolution of infection

Implant failure; No cases of implant failure in either groups . Knee stiffness ; In RN group: one case suffered from mild knee stiffness and was managed by physiotherapy ; In LP group: two cases; one suffered from mild and the other from moderate knee stiffness; both were managed by physiotherapy. Delayed union; In RN group: one case of delayed union managed by dynamization at the $14^{\text {th }}$ week and the fracture went to union after 8 weeks of dynamization ; In LP group: no cases of delayed union. Non-union ; In RN group: no cases of nonunion ; In LP group: one case of non-union (45 years male, diabetic and heavy smoker) that is scheduald for bone grafting. Knee pain ; In RN group: one case suffered from pain in the medial aspect of the knee due to locking screw protrusion on the medial aspect of the medial condyle, the case was managed by screw removal after healing process established ( $4^{\text {th }}$ month post-operative) ; LP group: one case complained of anterior knee pain with clicking sound with flexion and extension of the knee. Examination of the patient revealed tight lateral retinaculum and $\mathrm{X}$ ray showed no patellar tilt, then underwent 
physiotherapy and injection of local anesthetic and the pain improved after 2 months of physiotherapy.

End Results :- The retrograde nail was superior to locked plate in the aspect of , operative time with less operative blood loss ; less time to union ; minimal soft tissue injury and ability to mobilize patients early.

\begin{tabular}{|c|c|c|}
\hline & Mean \pm SD & P value \\
\hline $\begin{array}{c}\text { Retrograde } \\
\text { nail } \mathrm{n}=16\end{array}$ & $93.56 \pm 18.36$ & \\
\cline { 1 - 2 } $\begin{array}{c}\text { Locked } \\
\text { plate } \mathrm{n}=16\end{array}$ & $107.75 \pm 12.56$ & \\
\hline
\end{tabular}

Table 1: Distribution of cases according to operative time.

\begin{tabular}{|c|c|c|}
\hline & Mean \pm SD & P value \\
\cline { 1 - 2 } $\begin{array}{c}\text { Retrograde } \\
\text { nail } n=16\end{array}$ & $248.125 \pm 147.23$ & 0.003 \\
\cline { 1 - 2 } $\begin{array}{c}\text { Locked plate } \\
n=16\end{array}$ & $434.375 \pm 177.41$ & \\
\hline
\end{tabular}

Table 2: Distribution of cases according to blood loss in cc.

\begin{tabular}{|c|c|c|}
\hline & Mean \pm SD & P value \\
\hline $\begin{array}{c}\text { Retrograde nail } \mathrm{n}=15 \\
\text { (Range from 9 to 12 } \\
\text { weeks) }\end{array}$ & $11.4 \pm 1.92$ & 0.001 \\
\hline $\begin{array}{c}\text { Locked plate n=15 } \\
\text { (Range from 9 to 20 } \\
\text { weeks) }\end{array}$ & $15.8 \pm 3.37$ & \\
\hline
\end{tabular}

Table 3: Distribution of cases according to time to union.

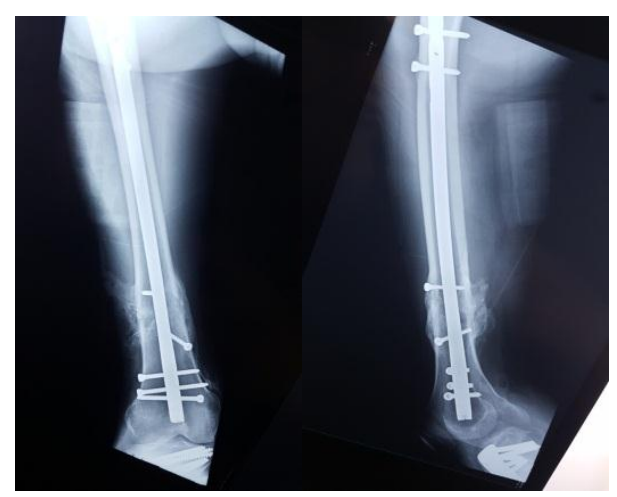

Fig. A: A case of Distal Femur Fracture Fixed By Retro grade nail After 6 months.

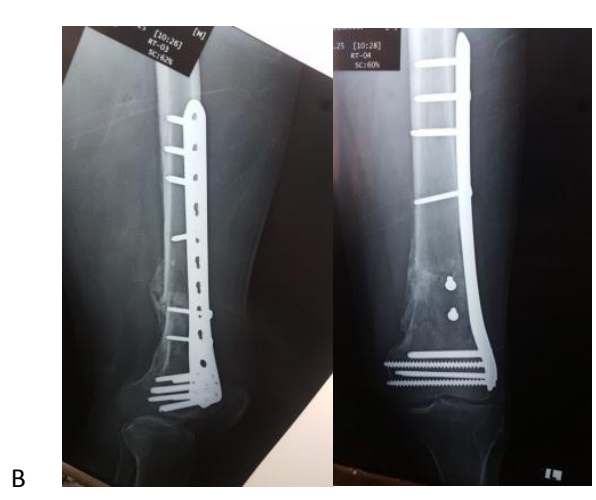

Fig. B: A case of Distal Femur Fracture Fixed By Locked Plate After 6 Months.

\section{DISCUSSION}

Distal femur fractures account for less than $1 \%$ of all fractures and about 3 to $6 \%$ of all femoral fractures ${ }^{[1]}$. Fractures of the distal femur have a bimodal distribution; younger people sustaining high-energy trauma, such as motor vehicle accidents or fall from height and older people after a minor fall, typically women, with osteoporosis.

The objective of this study was to compare retrograde intramedullary nail $(\mathrm{RN})$ versus distal femur locked compression plates (LP) in management of distal femur fractures (types A , C1 and $\mathrm{C} 2$ according to the AO/OTA classification) regarding functional and radiological outcome.

This study was conducted on 32 patients with distal femoral fractures; 16 cases treated with femoral retrograde interlocking nails (RN) and 16 cases treated with distal femoral locked compression plates (LP).

The patients were examined clinically and radiologically and were assessed for LEFS (lower extremity functional scale) ${ }^{[7]}$, Knee range of motion, time of union and complication rates.

Most important findings of our study:

operative time was statistically shorter in $\mathrm{RN}$ group ( $\mathrm{P}$ value of 0.017); Intraoperative blood loss was statistically significant between both groups, RN group had less blood loss (P value of 0.003) ; Time till union was also statistically shorter in th $\mathrm{RN}$ group ( $\mathrm{P}$ value of 0.001); LEFS was higher in $\mathrm{RN}$ group than LP group but it wasn't statistically significant (P value 0.95$)$; Knee range of motion also wasn't statistically significant in both flexion and extension (P value of 0.99 and 1 respectively); Post-operative Complications were higher in LP group but it wasn't statistically significant (P value 0.84 ).

We compared our results with the results of other studies that used the two techniques in management of distal femoral fractures. 
Hierholzer et al ${ }^{[8]}$, In a retrospective study from 2003 to 2008, They analyzed 115 patients with distal femur fracture (AO/OTA Type 33-A and C) who had been treated by retrograde IM nailing (59 patients) or LISS plating (56 patients). Henderson et al ${ }^{[9]}$, In their retrospective study, 174 distal femur fracture were reviewed to extract cases treated with retrograde IM nails (NAIL group, $n=12$ ). These were then individually matched to cases treated with locking plates with either open or closed techniques (Plate group, $\mathrm{n}=12$ ). Gao et al ${ }^{[10]}$, In a retrospective study of from January 2004 to March 2009, they reported 36 patients with extra-articular distal femoral fractures (AO/OTA Type 33-A). The patients were divided into two groups according to the treatment method, with 19 patients being treated by LP (in some cases LISS technique was used and open anatomic reduction was used in the other cases ) and 17 patients via RN. Markmiller et al ${ }^{[11]}$; in a prospective nonrandomized study included 32 patients they compared 16 patients treated with the Less Invasive Stabilization System (LISS) and 16 were treated with a distal femoral nail.

Comparison of the results between our study and the other studies : Demographic data and basic analysis :- In our study: the two groups were matched in the age, sex distribution, mode of trauma, comorbidities and smoking.This was in agreement with the other studies which had similar results regarding these parameters. However, the mean age in our study was lower than the other studies which was reflected on the mode of trauma with higher numbers of high energy impact. Operative time :- In our study there was significant difference $(\mathrm{p}$ value$=$ 0.017 ) in operative time between the two groups with mean time of 93.56 minutes for Retrograde Nail group and 107.75 minutes for LP group. Gao et al; There was no statistically significant difference in the mean operating time between the two groups ( $p=$ 0.106 ) with mean time of 87.4 minutes for Retrograde Nail group and 79.7 minutes for LP group. Markmiller et al; the mean time was 142 minutes for Retrograde Nail group and 155 minutes for LP group, it was statistically significant. The relatively long operative time in the LP group in our study may be attributed to use of open reduction rather than closed reduction which was used in most cases in Gao et al study (17 cases out of 19 plate case was manged by LISS technique) and all cases in Markmiller et al study. of data regarding operative time. Both Hierholzer et al and Henderson et al haven't commented on the operative time most propably due to the lack of data as both were retrospective studies. Intraoperative blood loss:- In our study there was statistically significant difference $(\mathrm{p}$ value $=0.003)$ regarding intra operative blood loss between the two groups with mean blood loss was 248.125 cc for Retrograde Nail group and 434.375 cc for LP group. Gao et al, The mean intraoperative blood loss was significantly higher in the RN group than in the LP group, with mean blood loss was $298 \mathrm{cc}$ for Retrograde Nail group and $200 \mathrm{cc}$ for LP group. The blood loss in RN group in our study was comparable to the other studies. However, the relative increase in blood loss in LP group in our study was due to use of open reduction technique. Postoperative hospital stay:-In our study; there was no significant difference ( $\mathrm{p}$ value $=0.39$ ) as regarding postoperative hospital stay in both groups. Mean hospital stay in RN group was 2.5 days and 2.8 days in LP group. We thought this was an important parameter as a good indicator for effect of the surgery on the general condition of the patient but other studies haven't commented on post-operative hospital stay. Time of union in weeks:- In our study; the mean time to union in Retrogade Nail group was 11.4 weeks while in LP group was 15.8 weeks with significant difference $(\mathrm{p}$ value $=0.001)$. Markmiller et al; the mean time to union in Retrogade Nail group was 14.6 weeks while in LP group was 13.8 weeks with significant difference. Gao et al; the time to union in the RN group and in the LP group was not statistically significant ( $\mathrm{p}$ value $=0.605$ ). Henderson et al; Significantly less periosteal callus formed in fractures stabilized with locking plates than with nails. Hierholzer et al; Time to union was not significantly different between the groups. The significance of union time in favor of $\mathrm{RN}$ group in our study is propably due to open reduction in LP group and periosteal stripping at the fracture site, also in the study of Henderson et al many cases of the LP group were managed by open technique so union time was shorter in RN group. That was not the case in the other studies were the plate group was managed using LISS technique which gave comparable results in union time with $\mathrm{RN}$ grouparding time to full union. Lower Extremity Function:- In our study; RN group had higher results according to LEFS score system but it wasn't statically significant ( $\mathrm{p}$ value $=0.95$ ) between both groups. Gao et al; There was no statistically significant difference in the HSS score $(p=0.406)$. Hierholzer et al: Statistical analysis of KOOS score results did not demonstrate significant differences between the groups for the accumulative result of KOOS and for function in daily living or function in sports and recreation. Markmiller et al; used Lysholm-Gillquist score to assess the lower extremity function with no statistically significant difference. The comparable results between our study and the other studies means that whatever the method (RN or LP) or the technique (open or closed) of fixation, when it is done properly, it gives good results. Range Of Knee Motion:- In our study; Retrograde Nail group had better knee ROM than LP group but it was not statistically significant ( $p$ value=0.44). Gao et al; There was statically insignificant difference regarding ROM of both groups $(\mathrm{P}=0.346)$ with the results being better with nailing. The results in our study and in the study of Gao et al was similar with better results in RN group, although it wasn't statistically significant it may be due to opening of the knee capsule in LP group with 
the open reduction technique. The other studies didn't comment on the knee range of motion as separate parameter and they depended on the different scoring systems as indicators for the functional outcome. Postoperative complication :- In most of the studies including ours there was no significant difference in the complication rate between both methods. However, in ours (although not significant) and in Gao, the healing disturbance was the most apparent. Study Strengths:- Comparative case study; Randomized controlled trial (RCT); The use of both a clinical evaluation system (Lower extremity functional scale) and radiological evaluation followup. Study Limitations:- Limited number of cases involved; The relatively short interval of follow-up (6 months); Use of open rather than closed reduction technique in LP group.

\section{CONCLUSION}

Based on this study; accepted outcome had been achieved with both methods compared with results of previous studies. However in our series nail showed more favorable outcome, less surgical morbidities and better rehabilitation as evident by :- Less blood loss during surgery ; Shorter operative time ; Shorter time required for union. However, both systems require precise preoperative planning and advanced surgical experience to reduce the risk of postoperative complications. Clinical outcome may largely depend on surgical technique rather than on the choice of implant. Recommendations for Further Research:- Multicenter studies with high numbers of patients would give better results ; Follow up period should be longer ; Use of closed rather than open technique in locked plate group.

\section{REFERENCES}

1. Coon, Matthew S., and Ben J. Best. "Distal Femur Fractures." In StatPearls [Internet]. StatPearls Publishing, 2019.

2. Court-Brown CM and Caesar B.: Epidemiology of adult fractures: a review. InjurIy 2006;37(8):691-7.

3. Walcher F, Frank J and Marzi I. Retrograde nailing of distal femoral fracture-clear and potential indications. European Journal of Trauma. 2000 Aug 1;26(4):155-68.
4. Mashru RP and Perez EA. Fractures of the distal femur: current trends in evaluation and management. Current Opinion in Orthopaedics. 2007 Feb 1;18(1):41-8.

5. Helfet DL and Lorich DG. Retrograde intramedullary nailing of supracondylar femoral fractures. Clinical orthopaedics and related research. 1998 May(350):80-4.

6. El-Kawy $S$, Ansara $S$, Moftah A, et al. Retrograde femoral nailing in elderly patients with supracondylar fracture femur; is it the answer for a clinical problem?. International orthopaedics. $2007 \mathrm{Feb}$ 1;31(1):83.

7. Binkley JM, Stratford PW, Lott SA, et al. North American Orthopaedic Rehabilitation Research Network. The Lower Extremity Functional Scale (LEFS): scale development, measurement properties, and clinical application. Physical therapy. 1999 Apr 1;79(4):371-83.

8. Hierholzer C, von Rüden C, Pötzel $\mathrm{T}$, et al. Outcome analysis of retrograde nailing and less invasive stabilization system in distal femoral fractures: A retrospective analysis. Indian $J$ Orthop 2011;45:243-50.

9. Henderson CE, Lujan $\mathrm{T}$, Bottlang $\mathrm{M}$, et al. Stabilization of distal femur frac-tures with intramedullary nails and locking plates: differences in callus formation. Iowa Orthop $J$ 2010;30:61-8.

10. Gao K, Gao W, Huang J, et al. Retrograde nailing versus locked plating of extra-articular distal fem-oral fractures: comparison of 36 cases. Med Princ Pract 2013;22:161-6.

11. Markmiller M, Konrad G and Südkamp N. Femur-:LISS and distal femoral nail for fixation of distal femoral fractures: are there differences in outcome and complications? Clin Orthop Relat Res 2004;426:252-7 . 